\title{
Criterio de diseño para el riego por gravedad con surcos cerrados
}

\author{
E. Vázquez Fernández y R. de la Cruz Gutiérrez \\ Instituto de Ingenieria, UNAM \\ Email: ernesfus.@servidor.unam.mx
}

(recibido: noviembre de 1997; aceptado: marzo de 1998 )

Resumen

\begin{abstract}
Se presenta un criterio de diseño para riego con surcos cerrados, basado en los resultados de simulación de un modelo numérico. Éste consiste en expresar en una ecuación de regresión lineal múltiple, el gasto que produce la máxima eficiencia de aplicación (gasto de diseño) en función de los parámetros más significativos, como longitud, pendiente, separación entre surcos, dosis, humedad inicial del suelo, coeficiente de rugosidad, conductividad hidráulica del suelo, y diferencia de tensiones y humedades del mismo; los tres primeros parámetros son de fácil medición; la dosis es un dato que se determina previamente; los últimos cuatro parámetros, que son los de mayor incertidumbre, se agrupan en un nuevo parámetro (no adimensional) característico de cada surco. Se incluye una ecuación para determinar este último parámetro no adimensional con la duración de la etapa de avance del agua, y otra para el tiempo de riego, así como un método gráfico alternativo. Un diseño con este criterio práctico puede ser empleado por el agricultor dentro de los intervalos dados para los parámetros mencionados y, con ello, lograr un uso eficiente del agua sin menoscabo de la producción.
\end{abstract}

\section{Abstract}

Based on numerical simulation, a criteria to design blocked furrows is presented. To develop it, the most significative parameters of the process such as length, slope, width, water deep, roughness coefficient, and some soil characteristics as initial moisture, and hydraulic conductivity and humidity tensions are related throught a multiple linear regression to determine the design discharge that causes the greatest efficiency. It is easy to determine, by direct measurement, the first three of these parameters. The water deep is one of the soil properties previously determined. In this paper, a new (dimensional) parameter is proposed to deal with them. This parameter is a characteristic of each furrow and an equation to determine it is included. Also it is given a formula to estimate the irrigation time. A chart-based procedure is given for practical purposes. We think that farmer could use these practical criteria, as long as he works in the recommended intervals for the quoted parameters, in order to achieve a more efficient water profit without reducing productivity.

\section{Introducción}

La necesidad de uso eficiente del agua de riego en México ha dado lugar a que las parcelas con surcos abiertos se conviertan en parcelas con surcos cerrados, lográndose un ahorro de agua y fertilizante al evitarse los coleos al final de los surcos. No obstante, conviene que los caudales de alimentación sean tales que se produzcan todavía mayores ahorros de agua, esto es, que sean los óptimos para obtener la máxima eficiencia de aplicación y aumentar, con el ahorro de agua, la superficie de riego.
La gran cantidad de parámetros que intervienen en el proceso de riego por surcos hace complejo su diseño, por lo que los investigadores buscan métodos simplificados que no incluyan a todos los parámetros sino sólo aquellos más significativos, empero, siempre habrá al menos uno que tenga un alto grado de incertidumbre, como es el caso de la conductividad hidráulica del suelo.

Entre los autores que han presentado métodos simplificados se encuentran Zierold (1992), Juana y Losada (1994) y Rendón et al (1995); además, Hernández et al (1995) quienes evaluaron el método de Zierold con resultados satisfactorios. El criterio de diseño de Juana y Losada es 
gráfico para surcos abiertos a partir de simulaciones numéricas con un modelo matemático de inercia nula y de la onda cinemática. Rendón et al reportan tablas de diseño para surcos cerrados en función de la textura del suelo, según los resultados de un modelo numérico hidrológico. El método que aquí se muestra considera los parámetros más significativos que intervienen en el proceso del riego por surcos, pero el agrupar a los de mayor incertidumbre en un parámetro no adimensional hace posible simplificar el diseño, si se realiza una prueba preliminar de riego, ya que se mide la duración de la etapa de avance del agua y se usan las ecuaciones de regresión. En el modelo numérico se emplea la fórmula teórica de Green y Ampt (Chu, 1978)

$$
V_{z}=K\left(1+\frac{\lambda}{Z}\right)
$$

donde $V z$ es la velocidad de infiltración vertical $(\mathrm{cm} / \mathrm{min})$, $K$ es la conductividad hidráulica del suelo $(\mathrm{cm} / \mathrm{min}), \lambda$ es el producto de la diferencia de tensiones y humedades antes y después de humedecerse el suelo $(\mathrm{cm})$, y $Z$ es la lámina de infiltración acumulada $(\mathrm{cm})$. Además, si esta velocidad se multiplica por el perímetro mojado de una sección transversal del surco y una longitud de tramo unitaria, se obtiene el gasto infiltrado por unidad de longitud de surco.

La geometría de los surcos empleados en las simulaciones numéricas es parabólica en tres de ellos - surcos Schwankl, Tarboton y Fangmeier-Ramsey tomados de Bautista y Wallender (1993) - ; en otros dos es triangular con ángulos en el vértice de 90 y $120^{\circ}$; en este último caso se empleó el modelo numérico para calibrar los parámetros de infiltración y rugosidad de un suelo arenoso en el Instituto de Ingeniería de la UNAM.

En los siguientes incisos se presentan las ecuaciones de regresión lineal que permiten obtener el gasto óptimo y el tiempo de riego. Al agrupar los parámetros de mayor incertidumbre es posible una representación gráfica de las ecuaciones para determinar, de manera alternativa, las variables de diseño (gasto y tiempo de riego); se presenta otra ecuación de regresión que da lugar a la estimación del parámetro característico del surco a partir de la duración de la etapa de avance del agua (tiempo que tarda en llegar el agua desde la cabecera del surco al extremo cerrado del mismo). Se incluye después un análisis de sensibilidad de los parámetros, una gráfica adimensional para estimar la eficiencia de aplicación en el caso de no alimentar el surco con el gasto óptimo, el procedimiento de cálculo cuya comprensión se amplía con ejemplos numéricos en un apéndice, y una breve comparación con otros métodos simplificados. Se concluye que las ecuaciones de regresión sustituyen al modelo numérico si se emplean dentro de los intervalos dados, pero que deben evaluarse en el campo, no obstante, haberse comprobado su validez en un surco corto de $50 \mathrm{~m}$.

\section{Ecuaciones y gráficas}

Los parámetros de mayor influencia para obtener el gasto óptimo en el proceso de riego por surcos (Vázquez, 1995) son la longitud del surco $(L s)$, la conductividad hidráulica del suelo $(K)$, la pendiente $(S o)$, el producto de la diferencia de tensiones y humedades antes y después de humedecerse el suelo $(\lambda)$, la rugosidad del surco $(n)$, la dosis $(\mathrm{Zm})$, la humedad inicial del suelo $(w)$ y la separación entre surcos $(D s)$. Los de mayor incertidumbre son la humedad inicial, la rugosidad, la conductividad hidráulica y la diferencia de tensiones del suelo; estos dos últimos son los parámetros de la ecuación de Green y Ampt, y la rugosidad del surco se valúa con el coeficiente de rugosidad de Manning. Si dichos parámetros de mayor incertidumbre se agrupan, se obtiene un nuevo parámetro (no adimensional) que interviene en dos ecuaciones de regresión lineal múltiple que permiten aproximarse al gasto óptimo, después de una primera prueba de riego donde se mida la duración de la etapa de avance del agua $(t a)$.

Con base en 729 simulaciones numéricas (27 arreglos diferentes de 27 surcos) del modelo numérico (Vázquez y Gracia, 1997), se obtuvieron las siguientes ecuaciones de regresión lineal múltiple para determinar el gasto óptimo $(Q o)$, el tiempo de riego $(T r)$ y el tiempo de avance del agua $(t a)$ :

$$
\frac{L_{s}}{Q_{0}}=24.66 P\left(\frac{S_{0} Z m^{0.1}}{D_{s}^{0.5}}\right)^{0.6},
$$

donde Qo está expresado en $l / s ; L s$, en $\mathrm{m} ; D s$, en $\mathrm{cm} ; S o$, en \% y $Z m$, en $\mathrm{cm}$. El coeficiente de correlación es de 0.96; el ajuste entre el modelo de regresión y el modelo numérico se muestra en la figura 1.

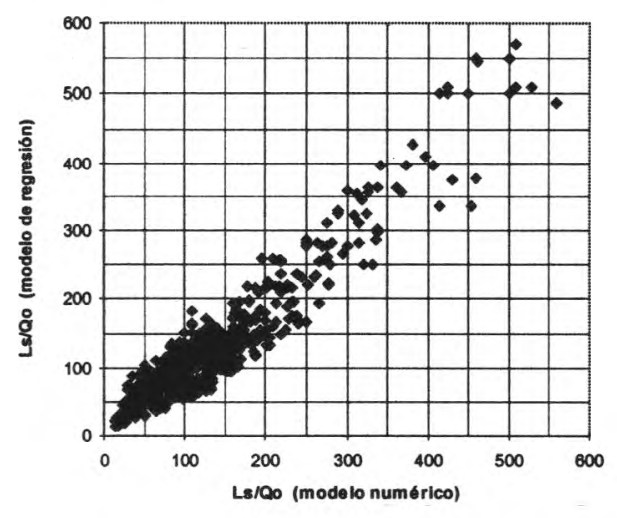

Figura 1. Ajuste de la ecuación 2 
Además,

$$
P=\frac{\mathrm{w}^{0.27}}{\mathrm{~K} n^{0.67} \lambda^{0.4}}
$$

donde $w$ está dado en $\mathrm{cm} ; K$, en $\mathrm{cm} / \mathrm{min} ; n$, en $\mathrm{s} / \mathrm{m}^{1 / 3} ; \mathrm{y} \lambda$, en $\mathrm{cm}$. En la ecuación 2, siempre que la eficiencia sea la máxima, existe una relación lineal entre la longitud y el gasto que también se obtiene para surcos abiertos (Vázquez, 1996) y que muestran Rendón et al (1995) para surcos cerrados; asimismo, hay una relación lineal de la conductividad hidráulica con el gasto, la cual se debe al empleo de la fórmula de Green y Ampt. Los intervalos empleados en los parámetros son los siguientes:

\begin{tabular}{cc}
\hline \hline Parámetro & Intervalo \\
\hline$L s$ & 40 a $400 \mathrm{~m}$ \\
$K$ & 0.0133 a $0.0833 \mathrm{~cm} / \mathrm{min}$ \\
$S o$ & 0.01 a $0.1 \%$ \\
$D s$ & 65 a $100 \mathrm{~cm}$ \\
$Z m$ & 5 a $15 \mathrm{~cm}$ \\
$w$ & 1 a $3 \mathrm{~cm}$ \\
$\lambda$ & 3 a $15 \mathrm{~cm}$ \\
$n$ & 0.03 a $0.012 \mathrm{~s} / \mathrm{m}^{1 / 3}$ \\
\hline
\end{tabular}

El intervalo del gasto óptimo $Q o$ es de 0.3 a $8.01 / \mathrm{s}$.

Los tiempos de riego fueron menores de 24 horas.

El intervalo de la conductividad hidráulica corresponde a una variación de la textura desde suelos franco-arcillosos a suelos arenosos (Cuenca, 1989).

La ecuación obtenida para el tiempo de riego con las 729 simulaciones numéricas es

$$
\operatorname{Tr}=0.0123\left(\frac{L s}{Q_{0}}\right)^{0.987}(D s Z m)^{0.722}
$$

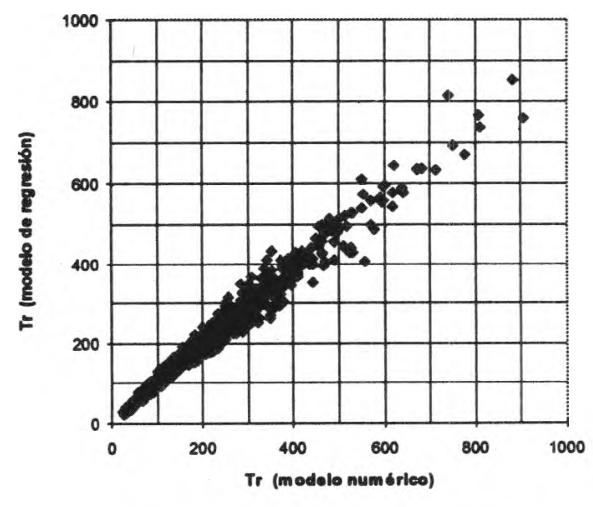

Figura 2. Ajuste de la ecuación 4 donde $\operatorname{Tr}$ queda expresado en min y los parámetros restantes, como se indica para la ecuación 2. El coeficiente de correlación es de 0.98. En la figura 2 se muestra el ajuste de los resultados de la ecuación con el modelo numérico. Aquí también se mantiene la relación lineal entre la longitud y el gasto, pero ya no tiene influencia el parámetro $P$.

De los parámetros incluidos en las ecuaciones anteriores, la dosis y la pendiente no influyen en la etapa de avance del agua, por lo que la ecuación obtenida con las 729 simulaciones numéricas al incluir la duración de la etapa de avance del agua es

$$
L s=10.68 P^{0.38}\left(\frac{Q o t a}{D s^{0.5}}\right)^{0.5},
$$

donde $\underline{t a}$ queda expresado en min y los parámetros restantes, como se indica para las ecuaciones 2 y 3 . El coeficiente de correlación es de 0.96 ; en la figura 3 puede verse el ajuste de la ecuación con el modelo numérico. Cabe hacer notar que en esta ecuación el produc to Qota es el volumen de ingreso al surco durante la etapa de avance del agua, y la relación con la longitud no es lineal.

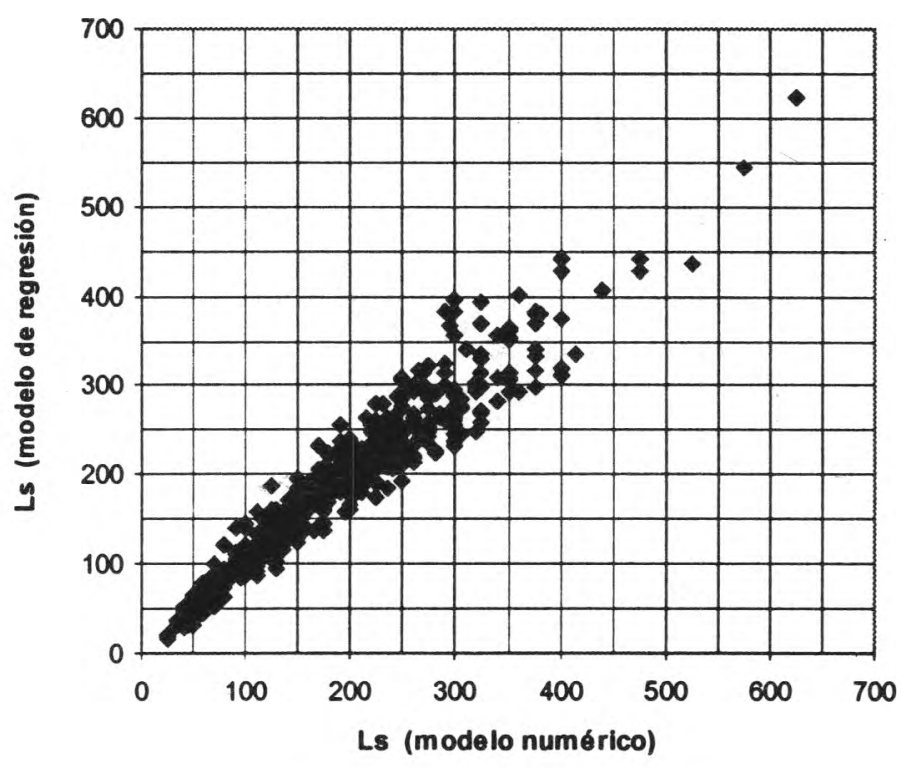

Figura 3. Ajuste de la ecuación 5

El arreglo de los parámetros en tres grupos para las ecuaciones 2,4 y 5 permite una representación gráfica, como se muestra en las figuras 4,5 y 6 , respectivamente. 


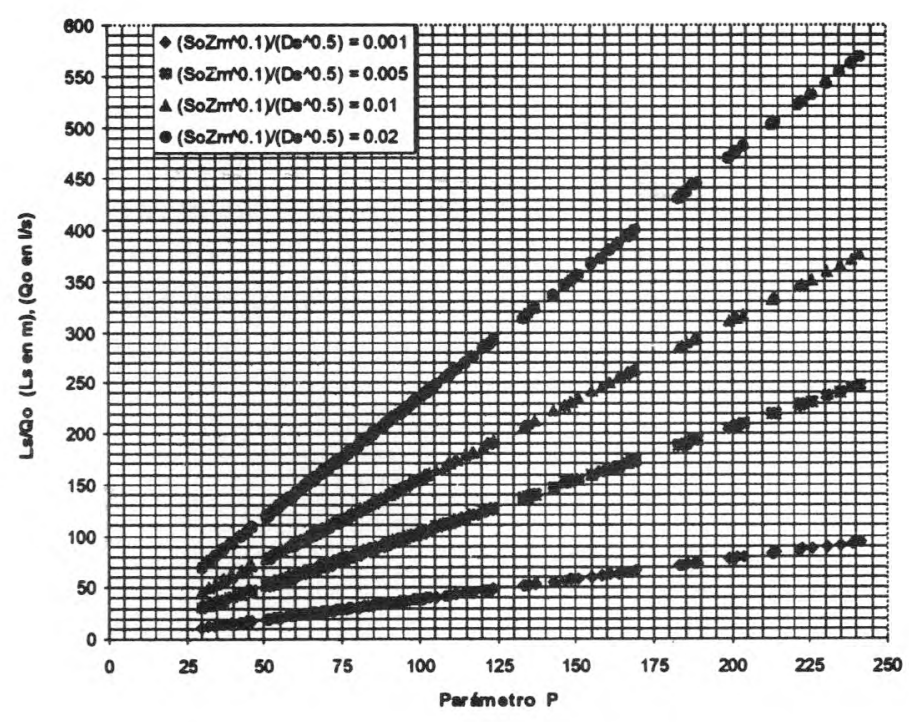

Figura 4. Gráfica para obtener el gasto óptimo

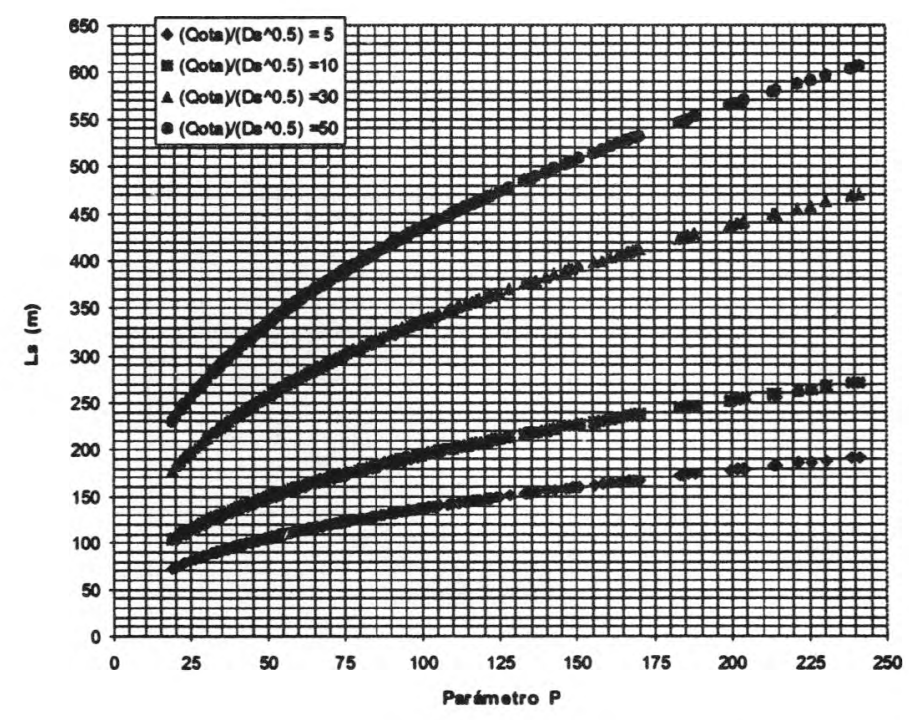

Figura 6. Gráfica para estimar el parámetro $P$

\section{Análisis de sensibilidad}

Por el valor de los exponentes en las ecuaciones 2 y 3 se concluye que los parámetros más significativos para el diseño del gasto óptimo son la longitud del surco $L s$, la conductividad hidráulica $K$, la pendiente $S o$, el coeficiente de rugosidad $n$, y que tienen menor influencia $D s, Z m, w$ y $\lambda$. Además, si de la ecuación 2 se acepta que el parámetro $P$ es característico para cada surco (y por ello se mantiene en la ecuación 5),

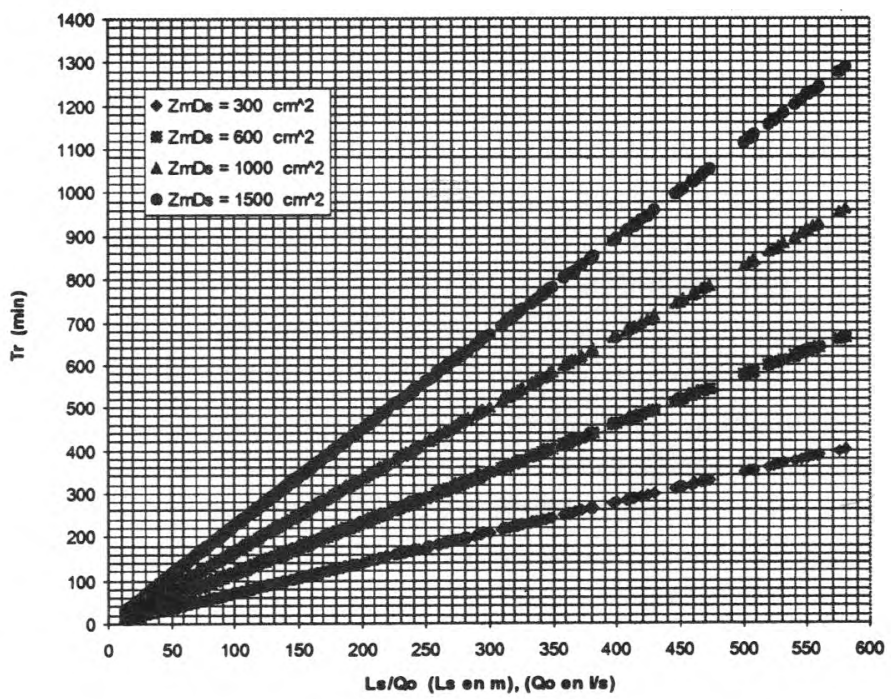

Figura 5. Gráfica para determinar el tiempo de riego

es posible mostrar en la figura 7 las curvas de eficiencias de aplicación $\eta$ contra relaciones de gastos $Q r / Q o$, donde $Q r$ es un gasto diferente al óptimo, para surcos que se identifican con el parámetro $P S o^{0.6}$. Esta figura es útil para estimar eficiencias de aplicación menores que la máxima cuando el gasto de alimentación al surco $(Q r)$ no es óptimo. Nótese que si $Q r / Q o=1$, la eficiencia es máxima; además, si $Q r / Q o<0.8$ para surcos con $P S o^{0.6}>10$ la curva se interrumpe porque el agua no alcanza a llegar al extremo cerrado del surco. Asimismo, la disminución de la eficiencia es menor cuando $Q r / Q o>1$, por lo que es recomendable que el gasto de diseño en el riego con surcos cerrados sea óptimo o mayor hasta en $1.25 Q o$, ya que la reducción de la eficiencia de aplicación no es excesiva (a lo más, de $5 \%$ ).

Además, las eficiencias más altas se obtienen para los valores más bajos de $P S o^{0.6}$, esto es, que las mayores eficiencias se obtendrán en surcos con pendientes suaves y suelos con textura arenosa (que tienen valores altos de $K$ ).

En la figura 4 se observa que para obtener el gasto óptimo, la influencia de la separación entre surcos y la pendiente es mayor a medida que crece $P$. Nótese que para valores bajos de $P$ la influencia de la separación entre surcos y la pendiente son menores; sobra decir que la influencia de la dosis en surcos cerrados es mínima.

En la ecuación 5 se presenta una relación lineal entre el gasto y la duración de la etapa de avance del agua, cuyo producto es el volumen de ingreso al surco; en la figura 6 se observa que la influencia de la duración de la etapa de avance y la separación entre surcos es mayor a medida que crece $P$, es decir, para suelos con textura franco arcillosa. 


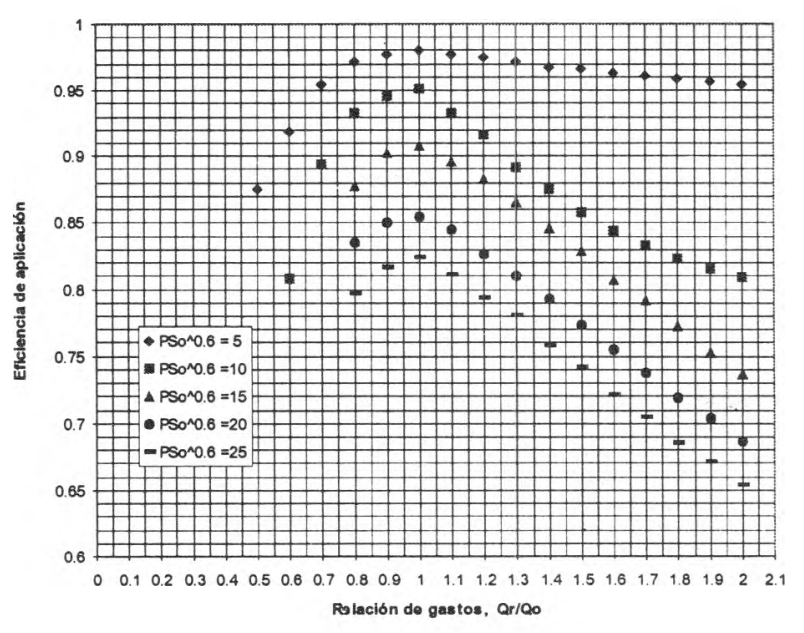

Figura 7. Gráfica para obtener las eficiencias de aplicación

\section{Procedimiento de cálculo}

a) Con las ecuaciones de regresión

Para un primer diseño se puede determinar la humedad inicial del suelo en lámina de agua $(w)$ dentro de un volumen limitado por $L s D s Z m$, estimarse el coeficiente de rugosidad de Manning ( $n$ ) con la textura del suelo, o determinarse, mediante pruebas con infiltrómetros, la conductividad hidráulica $(K)$ y el producto de la diferencia de teinsiones y humedades $(\lambda)$. Además de conocer $L s, D s, Z m$ y So, para emplear la ecuación 6 y determinar el gasto óptimo con la ecuación

$$
Q_{0}=\left(\frac{L s}{24.66 P}\right)\left(\frac{D s^{0.5}}{S o Z m^{0.1}}\right)^{0.6}
$$

El tiempo de riego ( $\operatorname{Tr}$ ) se calcula con la ecuación 4.

La eficiencia de aplicación ( $\eta$ ) se obtiene con la ecuación

$$
\eta=\frac{L s D s Z m}{(600) Q_{0} T r},
$$

donde $L s, D s, Z m, Q o$ y $T r$ tienen las unidades expresadas para las ecuaciones 2 y 4 .

b) Método gráfico

Con los mismos datos iniciales se calculan los grupos de parámetros de la figura 4 y se determina el gasto óptimo a través del cociente $L s / Q o$ obtenidoen el eje vertical.

El tiempo de riego se obtiene con la gráfica de la figura 5 .

Finalmente, la eficiencia de aplicación se calcula con la ecuación 7 .

\section{Aproximación al gasto óptimo}

Si después de un riego se mide la duración de la etapa de avance del agua y en la ecuación 5 se usa el gasto de alimentación $Q r$ en lugar del óptimo $Q o$, se obtiene que

$$
P=\left(\frac{L s}{10.68}\right)^{2.63}\left(\frac{D s^{0.5}}{\text { Qrta }}\right)^{1.32}
$$

Al sustituir este valor en la ecuación 6 se encuentra un nuevo gasto óptimo que sí es cercano al aplicado, por ejemplo con una diferencia de $0.1 \mathrm{l} / \mathrm{s}$ se acepta como el óptimo. Si no es así, debe aplicarse otro riego con el resultado obtenido mediante la ecuación 6 . Obsérvese que no es necesario conocer la humedad inicial del suelo, el coeficiente de rugosidad, los parámetros de la ecuación de Green y Ampt, ni la pendiente, pues la influencia de ellos se refleja en la duración de la etapa de avance del agua.

De manera gráfica, con el valor de $P S o^{0.6}$ y la relación $Q r / Q o$ es posible observar en la figura 6 la aproximación al gasto óptimo (cuando $Q r / Q o=1$ ).

En el apéndice se presentan tres ejemplos para ilus. trar el empleo de las ecuaciones y gráficas; en el primero de ellos se usan datos obtenidos en una prueba de riego efectuada en un surco construido en las instalaciones del Instituto de Ingeniería de la UNAM.

\section{Comparación con métodos simplificados}

El método de Zierold (1992) consiste en suponer que la forma de la distribución de la lámina de infiltración en el surco es trapecial, con una relación, cuando el gasto es el óptimo, $Z i / Z f=1.2$, donde $Z i$ y $Z f$ son las láminas de infiltración en la cabecera y al final del surco, respectivamen. te. Además, también cuando el gasto es óptimo se cumple la relación $T r / T f=1.2$ a 1.5 , donde $T r$ es el tiempo de riego, y $T f$ es el tiempo de infiltración en el extremo cerrado del surco, el cual se mide en el campo. Esta última relación es la más importante y es la que se usa para hacer el ajuste al diseño del riego.

Los resultados del modelo numérico empleado en este trabajo muestran que cuando el gasto es óptimo, la relación $T r / T f$ varía de 0.67 a 1.11 , es decir, al ser generalmente mayor el tiempo de infiltración en el extremo cerrado del surco, la lámina de infiltración ahí es mayor que la lámina de infiltración en la cabecera, esto es, se forma un trapecio diferente al supuesto por Zierold. En caso contrario, cuando el gasto es menor al óptimo se forma un trapecio similar al de Zierold, por lo que el gasto óptimo del método propuesto por este autor es menor que el del método aquí presentado. 
Una diferencia notable con el método de Rendón et al (1995) es que al emplear ellos un modelo hidrológico de balance de volumen, que sólo considera la ecuación de continuidad y no la dinámica en las ecuaciones de Saint-Venant (Walker y Skogerboe, 1987), no incluyen como parámetro la pendiente del surco. No obstante, en algunos casos, las diferencias en el gasto óptimo pueden ser menores de $25 \%$, como puede verse en el ejemplo 3 del apéndice.

\section{Conclusiones}

Se identifica un valor característico en el riego con surcos cerrados a través del parámetro no adimensional $P$ para determinar el gasto óptimo: en $P$ quedan incluidos los parámetros de mayor incertidumbre como son la humedad inicial del suelo, el coeficiente de rugosidad de Manning, la conductividad hidráulica y el producto de las diferencias de tensiones y humedades antes y después de humedecerse el suelo.

La longitud del surco y la conductividad hidráulica mantienen una relación lineal con el gasto, lo cual concuerda con la fórmula teórica de Green y Ampt empleada en el cálculo del gasto de infiltración vertical; sin embargo, cuando se incluye la duración de la etapa de avance del agua, la relación de la longitud con el gasto ya no es lineal, pero sí lo es el producto de esta duración con el gasto, es decir, el volumen alimentado al surco durante la fase inicial del riego.

Se obtiene un criterio de convergencia para hallar el gasto de diseño óptimo a través del conocimiento de la duración de la etapa de avance del agua al aplicar varios riegos, o sea, el método es autocorrectivo y equivale a calibrar los parámetros de mayor incertidumbre.

Se presentan curvas de eficiencias de aplicación para diferentes gastos de riego en surcos cerrados para valores extremos y medios, dentro de los intervalos empleados, del parámetro $P S o^{0.6}$. Estas curvas son útiles para estimar la reducción de la eficiencia de aplicación por no alimentar al surco con el gasto óptimo. Así, conviene aplicar el riego por gravedad con surcos cerrados, pues se alcanzan eficiencias máximas teóricas hasta de $95 \%$ en suelos arenosos con pendiente suave; además, el gasto de diseño debe encontrarse entre el óptimo y $25 \%$ mayor que el óptimo, para que la reducción de la eficiencia de aplicación máxima sea menor de $5 \%$.

Se dispone de una herramienta práctica para el diseño del riego por gravedad con surcos cerrados a través de las ecuaciones de regresión lineal (que sustituyen al modelo numérico) o las gráficas de las figuras 4,5 y 6 , siempre que se aplique dentro de los intervalos dados para cada parámetro. El criterio se comprueba con un ensayo realizado en un surco corto de $50 \mathrm{~m}$, pero debe ser evaluado en el campo para surcos más largos.

\section{Agradecimientos}

Al Dr. Jesús Gracia Sánchez por la revisión crítica del escrito.

\section{Referencias}

Bautista E. y Wallender W. W. (1993). Reliability of Optimized Furrow-Infiltration Parameters. Journal of Irrigation and Drainage Engineering, Vol. 119, No. 5.

Cuenca R. H. (1989). Irrigation System Design. Prentice Hall, New Jersey, USA.

Chu S. T. (1978). Infiltration During un Unsteady Rain. Water Resources Research, Vol. 14, No. 3.

Hernández S. R., Echeverría S. G. y Zavala G. E. (1995). Evaluación de dos metodologías para el diseño de riego por surcos. VI Congreso Nacional de Irrigación, Chapingo, México.

Juana L. y Losada H. (1994). Semejanza hidrodinámica en riegos por superficie. Ingeniería del Agua, Vol. 1, No. 1, Universidad Politécnica de Valencia, España.

Rendón P. L., Fuentes R. C. y Magaña S. G. (1995). Diseño simplificado del riego por gravedad. VI Congreso Nacional de Irrigación, Chapingo, México.

Vázquez E. (1995). Análisis de sensibilidad de las variables que intervienen en el proceso de riego por surcos. Ingeniería, Vol. XV, No. 3. UNAM, México.

Vázquez E. (1996). Diseño y revisión de surcos con riegos continuo y de corte posterior. Ingeniería del Agua, Vol. 3, No. 3. Universidad Politécnica de Valencia, España.

Vázquez E. y Gracia J. (1997). Calibración de parámetros de infiltración y rugosidad con un modelo numérico para riego con surcos cerrados. Ingeniería del Agua, Universidad Politécnica de Valencia, España. En revisión.

Walker W. R. y Skogerboe G. V. (1987). Surface Irrigation. Prentice Hall, New Jersey, USA.

Zierold R. L. (1992). Modernización del riego por surcos. Tercera Conferencia Regional Panamericana del ICID. Memorias Técnicas, Tomo II, Vol. II, Mazatlán, México.

\section{Lista de símbolos}

Ds = Separación entre surcos

$\mathrm{K}$ = Conductividad hidráulica del suelo en la fórmula de Green y Ampt

Ls = Longitud del surco

Qo $=$ Gasto óptimo de alimentación al surco

$\mathrm{Qr}=$ Gasto de alimentación al surco diferente al óptimo

So $=$ Pendiente longitudinal del surco

ta = Duración de la etapa de avance del agua

$\mathrm{Tr}=$ Tiempo de riego 
$\mathrm{Tf}=$ Tiempo de infiltración en el extremo cerrado del sur co en el método de Zierold

$V_{z}=$ Velocidad de infiltración vertical en la fórmula de Green y Ampt

Z = Lámina de infiltración acumulada en la fórmula de Green y Ampt

Zi = Lámina de infiltración en la cabecera del surco en el método de Zierold

Zf = Lámina de infiltración en el extremo cerrado del sur co en el método de Zierold

$\mathrm{Zm}=$ Dosis por infiltrar

$\eta=$ Eficiencia de aplicación

$\lambda=$ Producto de las diferencias de tensiones y humedades antes y después de humedecerse el suelo en la fórmu la de Green y Ampt

\section{Apéndice. Ejemplos numéricos}

1. En un ensayo realizado en el Instituto de Ingeniería de la UNAM sobre un surco de $50 \mathrm{~m}$ de longitud, con pendiente $0.1 \%$ y separación entre surcos de $90 \mathrm{~cm}$, se midió una duración de la etapa de avance de 6 min y un tiempo de riego de 26 min para infiltrar una dosis de 5 $\mathrm{cm}$. La eficiencia de aplicación fue de $92 \%$ para un gasto de $1.8 \mathrm{l} / \mathrm{s}$. No obstante que la eficiencia es mayor de $90 \%$, se desea saber si el gasto se aproxima al óptimo dado por las ecuaciones de regresión.

De la ecuación 8 el parámetro $P$ es:

$$
P=\left(\frac{50}{10.68}\right)^{263}\left(\frac{(90)^{0.5}}{(18)(6)}\right)^{132}=49
$$

Al sustituir este valor en la ecuación 6 se obtiene

$$
Q o=\left(\frac{50}{24.66(49)}\right)\left(\frac{(90)^{0.5}}{0.1(5)^{0.1}}\right)^{0.6}=0.58 \mathrm{l} / \mathrm{s}
$$

En un segundo ensayo con un gasto de $0.61 / \mathrm{s}$, se obtuvo una duración de la etapa de avance de $18 \mathrm{~min}$ y una eficiencia de aplicación de $94 \%$. Sin embargo, al calibrar los parámetros con el modelo numérico se calculó un valor $P=32.6<49$; esta diferencia se atribuye, más que a los gastos, a diferencias en la compactación del suelo, ya que antes de cada ensayo el suelo se compacta, lo que se refleja en los parámetros de infiltración de la ecuación de Green y Ampt.

2. Supóngase un surco con $L s=190 \mathrm{~m}, S o=0.03 \%, D s=$ $75 \mathrm{~cm}$, y valores estimados de $w=3 \mathrm{~cm}, K=0.023$ $\mathrm{cm} / \min , n=0.04$, y $\lambda=10 \mathrm{~cm}$. La dosis por infiltrar es $\mathrm{Zm}=10 \mathrm{~cm}$; y se desea calcular el gasto óptimo. a) Con las ecuaciones de regresión

De la ecuación 3

$$
P=\frac{(3)^{0.27}}{(0.023)(0.04)^{0.67}(10)^{0.4}}=200
$$

De la ecuación 6

$$
Q o=\left(\frac{190}{24.66(200)}\right)\left(\frac{(75)^{0.5}}{(0.03)(10)^{0.1}}\right)^{0.6}=1 \mathrm{l} / \mathrm{s}
$$

El tiempo de riego se calcula con la ecuación 4

$$
\operatorname{Tr}=0.0123\left(\frac{190}{1}\right)^{0.987}((75)(10))^{0.722}=260 \mathrm{~min}
$$

La eficiencia de aplicación se calcula con la ecuación 7

$$
\eta=\frac{(190)(75)(10)}{(600)(1)(260)}=0.91
$$

Esta eficiencia sólo puede alcanzarse en el riego por surcos cuando estos son cerrados.

b) Método gráfico

Con el valor calculado de $P$ y

$$
\frac{S o Z m^{0.1}}{D s^{0.5}}=\frac{0.03(10)^{0.1}}{(75)^{0.5}}=0.0044
$$

se obtiene, de la figura 4, $L s / Q o=190$, por lo que $Q o=1 \mathrm{l} / \mathrm{s}$, que es el mismo valor dado por las ecuaciones de regresión.

Para determinar el tiempo de riego con la gráfica de la figura 5, se tiene que $L s / Q o=190$ en el eje horizontal, y que

$$
Z m D s=(10)(75)=750 \mathrm{~cm}^{2}
$$

Al hacer una interpolación entre las rectas $Z m D s=$ 600 y $Z m D s=1000$, se obtiene un valor aproximado de $\operatorname{Tr}=260 \mathrm{~min}$ en el eje vertical, que es el calculado con la ecuación 4. Por tanto, la eficiencia de aplicación tam. bién es de 0.91 .

3. Supóngase ahora un surco con $L s=100 \mathrm{~m}, D s=75$ $\mathrm{cm}$, y $S o=0.1 \%$, donde se aplica un gasto $Q r=2.025$ $l / s$ para infiltrar una dosis $Z m=6 \mathrm{~cm}$ y se mide una duración de la etapa de avance $t a=37.1 \mathrm{~min}$. Se desea saber si gasto es óptimo de acuerdo con las ecuaciones 6 y 8 . 
Según la ecuación 8 , la estimación del parámetro característico del surco es

$$
P=\left(\frac{100}{10.68}\right)^{263}\left(\frac{(75)^{0.5}}{(2025)(37.1)}\right)^{132}=21
$$

$\mathrm{Al}$ sustituir este valor en la ecuación 6 se tiene que

$$
Q o=\left(\frac{100}{24.66(21)}\right)\left(\frac{(75)^{0.5}}{(0.1)(6)^{0.1}}\right)^{0.6}=25 \mathrm{l} / \mathrm{s}
$$

Este valor tiene un porcentaje de error de $23 \%$, si se acepta como gasto óptimo el de $2.025 \mathrm{l} / \mathrm{s}$ que se tomó de la tabla de diseño dada por Rendón et al (1995) para una textura del suelo franco arenosa. El intervalo dado por los autores para la pendiente es de 0.05 a $0.5 \%$. Si se considera una pendiente So $=0.144 \%$ en la última ecuación ( $4.4 \%$ arriba del límite superior en la ecuación 2), el resultado obtenido es el mismo de los autores, pero si el surco tuviera otro valor más bajo de la pendiente, la diferencia en el gasto sería mayor, por lo que se confirma que la influencia de la pendiente en la ecuación de regresión para el diseño del gasto óptimo es importante. Asimismo, se ha desempeñado como especialista en ingeniería hidráulica en los sectores público y privado. Actualmente, labora en el Instituto de Ingeniería, UNAM.

Rafael de la Cruz Gutiérrez. Egresado de la Universidad Autónoma de Chiapas, ha laborado como profesor en la Universidad del Valle de Grijalva, como topógrafo de la SCT, y constructor de vivienda de interés social. Actualmente, es estudiante de la maestría en ingeniería hidráulica en la DEPFI, UNAM. 